\title{
IMPROVEMENT OF SWEET PEPPER (Capsicum annuUm L.) PRODUCTIVITY USING SOME ANTIOXIDANTS UNDER SALINITY CONDITIONS OF SOUTH SINAI \\ I. MORPHOLOGICAL GROWTH CHARACTERS
}

\author{
Sozan F. Rashed ${ }^{1 *}$, M.I. Mahmoud ${ }^{2}$, E.M. El-Tantawy ${ }^{2}$, \\ Hosna A. Fouad ${ }^{1}$ and A.I. El-Kassas ${ }^{2}$
}

1. Cent. Desert Res., Cairo, Egypt.

2. Dept. Plant Prod., Fac. Environ. Agric. Sci., Arish Univ., Egypt.

\begin{abstract}
Two field experiments were conducted during summer seasons of each 2012 and 2013 in the Ras-Suder Research Station, South Sinai, Desert Research Center. The main object of this research was studying the effect of some antioxidant treatments on morphological growth characters of sweet pepper plant (Capsicum annuum L.) hybrid cultivar "Sonar". Aqueous solutions of antioxidants were used as foliar spray; viz., ascorbic acid (Vitamin C), oxalic acid, salicylic acid and, tocopherol (Vitamin E) within four different concentrations of these antioxidants $(0.0,200,400$ and $600 \mathrm{ppm})$ were applied at 20, 40 and 60 days after transplanting. Obtained results reflected that the highest values of growth parameters expressed as plant height, number of branches and leaves per plant, fresh and dry weight per plant and leaf area per plant as well as reading total chlorophyll were significantly affected by antioxidants, the highest values were recorded spraying with salicylic acid and ascorbic acid at $400 \mathrm{ppm}$.
\end{abstract}

Key words: Ascorbic acid, oxalic acid, salicylic acid, tocopherol, growth, sweet pepper.

\section{INTRODUCTION}

Sweet Pepper (Capsicum annuum L.) is an important vegetable crop, not only because of its economic importance, but also for the nutritional value of its fruits, mainly due to the fact that it is an excellent source of natural colures and antioxidant compounds (Howard et al, 2000). Pepper fruits considered an excellent source of bioactive nutrients such as carotenoids, vitamin $\mathrm{C}$ and phenolics compounds (Navarro et al., 2006).

The sequence of events in the plant tissue subjected to salinity stress increased the production of Reactive Oxygen Species

\footnotetext{
* Corresponding author: Tel.: +201069294723

E-mail address: suzanrashed123@yahoo.com
}

(ROS) and of oxidized target molecules, increases in the levels of anti-oxidative systems and antioxidants also increased scavenging capacity for ROS, resulting in tolerance against the salinity stress (Mano, 2002). Ascorbic acid is an important antioxidant has been shown to protect plants. Vitamin $\mathrm{C}$ led to increase nucleic acid content especially RNA. Vitamin C functions as antioxidant, an enzyme factor and as growth regulating factor. It plays an important role in different processes, including photosynthesis, photo protection, cell wall growth and cell expansion resistance to environmental stresses of, synthesis of ethylene, gibberellins anthocyanins and hydroxyl proline 
(Nicholas and Wheeler 2000). Smirnoff and Wheeler (2000) reported that ascorbic acid is an abundant component of plants. It reaches a concentration of over $20 \mathrm{mM}$ in chloroplasts and occurs in all cell compartments including cell wall.

Oxalic acid is a common constituent of plant, and several species accumulate high levels of the simplest dicarboxylic acid. The most striking chemical property of oxalic acid is its strong chelating ability with multivalent cations. Recently, oxalic acid application has received much attention in relation to induced disease systemic resistance and its antioxidant capability (Zhang et al., 1999; Malencic et al., 2004).

Salicylic acid has numerous functions, particularly the inhibition of germination and growth, interference with root absorption, reduced transpiration and leaf abscission. Salicylic acid is widely present in plant and functions as a hormonal mediator of the systemic acquired resistance response. Thus, it presents in a large scale of fruits, vegetable, herbs and spices of dietary relevance.

Salicylic acid as plant phenolic is now considered as a hormone-like endogenous regulator, and has a role in the defence mechanisms against biotic and unbiotic stressors has been well documented (Yalpani et al., 1994). SA potentials the generation of reactive oxygen species in photosynthetic tissues during salt and osmotic stresses (Borsani et al., 2001). It plays a vital role in plant growth, ion uptake and transport. Tocopherol (Vitamin E) is an essential vitamin for humans and animals, it is exclusively synthesized in photosynthetic organisms (DellaPenna, 2005). Phytoregulator compounds (Vitamin E) suggested to elevate and tolerate the adverse effects of biotic and unbiotic stresses such as moisture and salt stress on plant growth and yield (Demiral and Turkan 2005; Raja Babo et al., 2005).
The main objective of this research was to study the effect of applying some antioxidants on sweet pepper (Capsicum annuum L., hybrid cultivars "Sonar") to alleviate the harmful effect of salinity under South Sinai conditions.

\section{MATERIALS AND METHODS}

A field experiment was carried out during the summer season of each 2012 and 2013 at The Experimental Farm, Research Station Ras Suder, South Sinai Desert Research Center. The main objective of this research was to study the effect of some antioxidants and their concentrations on increasing resist of pepper plant to alleviate harmful effect of salinity which reflected on morphological growth parameters of sweet pepper plants under sandy loam soil conditions using drip-irrigation system.

The mechanical and chemical analyses of the experimental soil are presented in Table 1. The soil analysis was carried out according to Richards (1954), Black and Editor (1965) and Jackson (1967). The hybrid cultivar "Sonar" was used in this study. Seeds of sweet pepper were sown on $10^{\text {th }}$ February in seedling trays under plastic green house conditions. The transplants were set up into the field on $1^{\text {st }}$ of April in both of 2012 and 2013 seasons.

Seedlings were transplanted besides dripper lines, the distance between every two dripper lines were $100 \mathrm{~cm}$. The distance between plants in the same line was $50 \mathrm{~cm}$. The plot area was $12 \mathrm{~m}^{2}$ (1m width $\mathrm{x} 12 \mathrm{~m}$ length). The fertigation method was used in the experiment by water pumped from a well; the analysis of irrigation water is presented in Table 2.The experiment included 16 treatments which were all combinations between 4 antioxidants (ascorbic acid, oxalic acid, Salicylic acid, and Tocopherol) and 4 concentrations (Control, $200 \mathrm{ppm} \mathrm{1}^{-1}, 400 \mathrm{ppm} \mathrm{l}^{-1}$, and 600 ppm 1 $\left.{ }^{-1}\right)$. 
Table (1): Chemical properties of the experimental soil.

\begin{tabular}{|c|c|c|c|c|c|c|c|c|c|c|c|c|c|}
\hline \multirow{3}{*}{$\begin{array}{c}\text { Dept } \\
\text { h } \\
(\mathbf{c m})\end{array}$} & \multirow{3}{*}{ pH } & \multirow{3}{*}{$\begin{array}{c}\text { E.C } \\
\mathrm{dS} / \mathrm{m}^{2-}\end{array}$} & \multicolumn{7}{|c|}{$\begin{array}{l}\text { Saturation soluble extract } \\
\qquad(\mathrm{mg} / \mathbf{1 0 0 g})\end{array}$} & \multicolumn{4}{|c|}{$\begin{array}{l}\text { Total nutrients } \\
\qquad\left(\mathrm{mg} \mathrm{Kg}^{-1}\right)\end{array}$} \\
\hline & & & \multicolumn{3}{|c|}{ Cations } & \multicolumn{4}{|c|}{ Anions } & \multirow{2}{*}{$\mathbf{N}$} & \multirow{2}{*}{$\mathbf{P}$} & \multirow{2}{*}{$\mathbf{K}$} & \multirow{2}{*}{$\mathbf{F e}$} \\
\hline & & & $\mathrm{Ca}^{++}$ & $\mathbf{M g}^{++}$ & $\mathrm{Na}^{+}$ & $\mathrm{Co}_{3}{ }^{-}$ & $\mathrm{HCO}_{3}$ & $\mathrm{So}_{4}{ }^{--}$ & $\mathrm{Cl}^{-}$ & & & & \\
\hline $0-30$ & 7.7 & 8.65 & 24.5 & 5.2 & 56.5 & 0.00 & 6.0 & 19.0 & 61.5 & 26.0 & 5.1 & 51.5 & 4.2 \\
\hline $30-60$ & 7.9 & 7.35 & 16.8 & 3.8 & 52.4 & 0.00 & 3.5 & 21.0 & 49.0 & 23.5 & 3.4 & 35.3 & 3.4 \\
\hline
\end{tabular}

Table (2): Chemical analysis of irrigation water.

\begin{tabular}{|c|c|c|c|c|c|c|c|c|c|}
\hline \multirow{3}{*}{ pH } & \multirow{3}{*}{$\begin{array}{c}E C \\
d S / \mathbf{m}^{2-}\end{array}$} & \multicolumn{8}{|c|}{ Soluble ions (meq. $1^{-1}$ ) } \\
\hline & & \multicolumn{4}{|c|}{ Cations } & \multicolumn{4}{|c|}{ Anions } \\
\hline & & $\mathrm{Ca}^{++}$ & $\mathrm{Mg}^{++}$ & $\mathrm{Na}^{+}$ & $\mathbf{K}^{+}$ & $\mathrm{Cl}^{-}$ & $\mathrm{HCO}_{3}^{-}$ & $\mathrm{CO}_{3}{ }^{--}$ & $\mathrm{SO}_{4}^{-}$ \\
\hline 7.77 & 7.85 & 20.5 & 8.6 & 48.89 & 0.35 & 57.5 & 5.0 & 0.0 & 16.2 \\
\hline
\end{tabular}

The antioxidants were applied as folair spraying on sweet pepper plants three times at 20, 40 and 60 days after transplanting. The design of the experiment in the field was split plot with three replications. Antioxidants were arranged randomly in the main plots, while their concentrations were distributed randomly in sub plots. Conventional culture practices were done as needed and were similar to those used in commercial pepper production in the open field in South Sinai region.

\section{Data recorded}

Three plants were randomly taken from each experimental unit. Vegetative growth parameters were recorded three times $(30$, 50 and, 70 days after transplanting), the following parameters were recorded: Plant height $(\mathrm{cm})$, Number of branch/plant, number of leaves/plant, Branch fresh weight/ plant $(\mathrm{g})$, Leaves fresh weight/ plant $(\mathrm{cm})$, Branch dry weight/plant (g), Leaf dry weight/ plant $(\mathrm{g})$, Leaf area per plant $\left(\mathrm{cm}^{2}\right)$, and Total chlorophyll reading (SPAD) chlorophyll was determined in the fourth leaf from pepper plant top using a digital chlorophyll meter, model Minolta Chlorophyll Meter SPAD-502, (Manufactured by Minolta Company, Japan).

\section{Statistical analysis}

Statistical analysis of the obtained data was carried out according to Statistical analysis of variance according to Snedecor and Cochran (1980). Duncan s multiple range tests was used for comparison among means (Duncan's, 1958).

\section{RESULTS AND DISCUSSION}

\section{Effect of antioxidants}

\section{Plant height and number of both branches and leaves}

Concerning the effect of antioxidants data in Table 3 show that application of antioxidant materials; viz, ascorbic acid, oxalic acid, salicylic acid and tocopherol affected significantly all studied traits at all 
Table (3): Effect of antioxidant on plant height, number of branches and number of leaves of sweet pepper during 2012 and 2013 seasons.

\begin{tabular}{|c|c|c|c|c|c|c|c|c|c|}
\hline \multirow{3}{*}{ Character } & \multicolumn{3}{|c|}{ Plant height (cm) } & \multicolumn{3}{|c|}{ No. of branches/ plant } & \multicolumn{3}{|c|}{ No. of leaves/plant } \\
\hline & \multicolumn{9}{|c|}{ Days after transplanting } \\
\hline & 30 & 50 & 70 & 30 & 50 & 70 & 30 & 50 & 70 \\
\hline & \multicolumn{9}{|c|}{ First season (2012) } \\
\hline Ascorbic acid & $21.10 \mathrm{~b}$ & $36.97 b$ & $43.91 \mathrm{a}$ & $2.15 \mathrm{ab}$ & $5.33 b$ & $10.63 b$ & $35.94 \mathrm{a}$ & 67.72ab & $98.26 \mathrm{a}$ \\
\hline Oxalic acid & $18.97 \mathrm{c}$ & $31.06 \mathrm{c}$ & $37.80 \mathrm{c}$ & $1.79 \mathrm{~b}$ & $4.45 \mathrm{c}$ & $9.51 \mathrm{c}$ & $32.47 \mathrm{~b}$ & $62.64 \mathrm{c}$ & $82.32 \mathrm{c}$ \\
\hline Salicylic acid & $22.61 \mathrm{a}$ & $39.19 a$ & $45.62 \mathrm{a}$ & $2.40 \mathrm{a}$ & $5.79 \mathrm{a}$ & $11.12 \mathrm{a}$ & $36.31 \mathrm{a}$ & $69.87 \mathrm{a}$ & $99.18 \mathrm{a}$ \\
\hline \multirow[t]{2}{*}{ Tocopherol } & $20.17 \mathrm{bc}$ & $35.96 \mathrm{~b}$ & $41.44 \mathrm{~b}$ & $1.90 \mathrm{ab}$ & $5.42 \mathrm{~b}$ & $9.43 \mathrm{c}$ & $33.66 \mathrm{~b}$ & $65.98 \mathrm{~b}$ & $89.18 b$ \\
\hline & \multicolumn{9}{|c|}{ Second season (2013) } \\
\hline Ascorbic acid & $21.78 \mathrm{~b}$ & $37.84 \mathrm{~b}$ & $45.45 b$ & $2.47 \mathrm{a}$ & $5.58 \mathrm{a}$ & $10.88 \mathrm{a}$ & $37.15 \mathrm{ab}$ & $70.89 b$ & $102.98 \mathrm{a}$ \\
\hline Oxalic acid & $19.26 \mathrm{c}$ & $32.17 \mathrm{c}$ & $38.43 \mathrm{~d}$ & $1.92 \mathrm{~b}$ & $4.59 \mathrm{~b}$ & $9.42 \mathrm{~b}$ & $32.75 \mathrm{c}$ & $65.31 \mathrm{c}$ & $84.87 \mathrm{c}$ \\
\hline Salicylic acid & $23.16 \mathrm{a}$ & $40.21 \mathrm{a}$ & $47.31 \mathrm{a}$ & $2.50 \mathrm{a}$ & $5.94 \mathrm{a}$ & $11.34 \mathrm{a}$ & $38.02 \mathrm{a}$ & $75.69 \mathrm{a}$ & $106.59 \mathrm{a}$ \\
\hline Tocopherol & $20.96 \mathrm{~b}$ & $36.79 \mathrm{~b}$ & $42.86 \mathrm{c}$ & $1.96 \mathrm{~b}$ & $5.57 \mathrm{a}$ & $9.65 \mathrm{~b}$ & $34.81 \mathrm{bc}$ & $70.42 b$ & $93.66 \mathrm{~b}$ \\
\hline
\end{tabular}

dates in both seasons. The highest values were recorded with application of salicylic acid followed by ascorbic acid and tocopherol, while the lowest value was recorded with application of oxalic acid.

\section{Fresh and dry weight ${ }^{\mathrm{s}}$ of plant}

Data presented in Table 4 show significant differences among antioxidants for fresh and dry weight of branches and leaves of pepper plants. In this respect, application of Salicylic acid had the highest values for all traits followed by ascorbic acid at 30, 50 and 70 days after transplanting in both growing seasons.

\section{Leaf area and total chlorophyll}

Data in Table 5 show significant effects for antioxidants on leaf area / plant of sweet pepper. In this respect, application of salicylic acid followed by ascorbic acid had the highest value for leaf area at all sampling dates in both growing seasons. For total chlorophyll (SPAD)' data in the same table show significant effects for antioxidants.

Application of ascorbic acid, salicylic acid and tocopherol recorded the best values in both season. At 70 days from transplanting in the first season the salicylic acid recorded the highest value for total chlorophyll. Whereas, oxalic acid recorded the lowest value for each of leaf area and total chlorophyll in all dates at both seasons.

\section{Effect of antioxidants concentration}

\section{Plant height and number of both branches and leaves}

As regard to the effect of concentrations of antioxidants, it was found that 400 or $200 \mathrm{ppm}$ had the highest significant value in both seasons followed by $600 \mathrm{ppm}$ and the lowest values were obtained with control treatments for all studied traits; i.e., plant height, number of branches and number of leaves per plant Table 6 . 
Table (4): Effect of antioxidant on fresh and dry weight of both branches and leaves of sweet pepper during2012 and 2013 seasons.

\begin{tabular}{|c|c|c|c|c|c|c|c|c|c|c|c|c|}
\hline \multirow{4}{*}{ Character } & \multicolumn{6}{|c|}{ Fresh weight (g) } & \multicolumn{6}{|c|}{ Dry weight (g) } \\
\hline & \multicolumn{3}{|c|}{ Branches } & \multicolumn{3}{|c|}{ Leaves } & \multicolumn{3}{|c|}{ Branches } & \multicolumn{3}{|c|}{ Leaves } \\
\hline & \multicolumn{12}{|c|}{ Days after transplanting } \\
\hline & 30 & 50 & 70 & 30 & 50 & 70 & 30 & 50 & 70 & 30 & 50 & 70 \\
\hline & \multicolumn{12}{|c|}{ First season (2012) } \\
\hline Ascorbic acid & $6.36 b$ & $24.03 b$ & $59.05 \mathrm{~b}$ & $10.56 \mathrm{~b}$ & $42.01 \mathrm{~b}$ & $71.23 b$ & $1.87 \mathrm{~b}$ & $5.82 \mathrm{a}$ & $12.21 b$ & $2.25 \mathrm{~b}$ & $6.06 \mathrm{~b}$ & $12.88 \mathrm{~b}$ \\
\hline Oxalic acid & $4.69 d$ & $20.37 d$ & $44.00 \mathrm{~d}$ & $9.28 \mathrm{c}$ & $35.12 \mathrm{c}$ & $59.62 d$ & $1.43 \mathrm{~d}$ & $4.39 \mathrm{c}$ & $10.76 \mathrm{~d}$ & $1.55 \mathrm{~d}$ & $4.66 \mathrm{~d}$ & $10.33 d$ \\
\hline Salicylic acid & $6.71 \mathrm{a}$ & $26.53 \mathrm{a}$ & $60.76 \mathrm{a}$ & $11.15 \mathrm{a}$ & $45.87 \mathrm{a}$ & $74.90 \mathrm{a}$ & $2.04 \mathrm{a}$ & $6.13 \mathrm{a}$ & $12.67 \mathrm{a}$ & $2.53 \mathrm{a}$ & $6.81 \mathrm{a}$ & $13.65 \mathrm{a}$ \\
\hline \multirow[t]{2}{*}{ Tocopherol } & $5.83 \mathrm{c}$ & $22.35 \mathrm{c}$ & $54.84 \mathrm{c}$ & $9.46 \mathrm{c}$ & $40.35 b$ & $67.45 \mathrm{c}$ & $1.68 \mathrm{c}$ & $5.02 \mathrm{~b}$ & $11.56 \mathrm{c}$ & $1.92 \mathrm{c}$ & $5.73 \mathrm{c}$ & $11.74 \mathrm{c}$ \\
\hline & & \multicolumn{11}{|c|}{ Second season( 2013) } \\
\hline Ascorbic acid & $6.71 \mathrm{a}$ & $24.41 b$ & $60.08 \mathrm{a}$ & $10.96 \mathrm{~b}$ & 42.72 & $73.12 b$ & $1.93 \mathrm{~b}$ & $5.89 \mathrm{~b}$ & $12.41 \mathrm{~b}$ & $2.36 b$ & $6.27 \mathrm{~b}$ & $13.42 b$ \\
\hline Oxalic acid & $4.71 \mathrm{c}$ & $20.93 c$ & $44.92 \mathrm{c}$ & $9.53 \mathrm{c}$ & $36.44 \mathrm{c}$ & $60.69 d$ & $1.47 \mathrm{~d}$ & $4.41 d$ & $10.92 d$ & $1.60 \mathrm{~d}$ & $4.75 d$ & $10.54 d$ \\
\hline Salicylic acid & $6.88 \mathrm{a}$ & $26.80 \mathrm{a}$ & $61.35 \mathrm{a}$ & $11.81 \mathrm{a}$ & $46.75 a$ & $76.34 \mathrm{a}$ & $2.16 \mathrm{a}$ & $6.20 \mathrm{a}$ & $12.88 \mathrm{a}$ & $2.69 \mathrm{a}$ & $7.03 \mathrm{a}$ & $13.78 \mathrm{a}$ \\
\hline Tocopherol & $6.08 b$ & $22.55 b c$ & $55.51 \mathrm{~b}$ & $9.86 \mathrm{c}$ & $41.84 b$ & $67.84 \mathrm{c}$ & $1.79 \mathrm{c}$ & $5.01 \mathrm{c}$ & $11.74 \mathrm{c}$ & $1.98 \mathrm{c}$ & $6.03 c$ & $12.28 \mathrm{c}$ \\
\hline
\end{tabular}

Values having the same alphabetical letter (s) did not significantly differ at 0.05 level of significance, according to Duncan's multiple range test.

Table (5): Effect of antioxidant on leaf area and total chlorophyll reading (SPAD) of sweet pepper during 2012 and 2013 seasons.

\begin{tabular}{|c|c|c|c|c|c|c|}
\hline \multirow[b]{3}{*}{ Treatments } & \multicolumn{3}{|c|}{$\begin{array}{c}\text { Leaf area/plant } \\
\left(\mathrm{cm}^{2}\right)\end{array}$} & \multicolumn{3}{|c|}{$\begin{array}{c}\text { Total chlorophyll reading } \\
\text { (SPAD) }\end{array}$} \\
\hline & \multicolumn{6}{|c|}{ Days after transplanting } \\
\hline & 30 & 50 & 70 & 30 & $\mathbf{5 0}$ & 70 \\
\hline & \multicolumn{6}{|c|}{ First season (2012) } \\
\hline Ascorbic acid & $338.1 \mathrm{ab}$ & $1631.8 b$ & $3142.8 b$ & $69.51 \mathrm{ab}$ & $71.03 \mathrm{a}$ & $71.46 b$ \\
\hline Oxalic acid & 277.1c & $1327.2 \mathrm{c}$ & $2101.5 \mathrm{c}$ & $67.97 \mathrm{~b}$ & $66.39 b$ & $67.70 \mathrm{~d}$ \\
\hline Salicylic acid & $371.3 \mathrm{a}$ & $1822.4 \mathrm{a}$ & $3517.1 \mathrm{a}$ & $70.57 \mathrm{a}$ & $72.10 \mathrm{a}$ & $72.30 \mathrm{a}$ \\
\hline Tocopherol & $322.9 b$ & $1512.4 b$ & $2853.2 b$ & $69.66 \mathrm{ab}$ & $69.53 \mathrm{ab}$ & $70.52 \mathrm{c}$ \\
\hline \multicolumn{7}{|c|}{ Second season (2013) } \\
\hline Ascorbic acid & $359.4 \mathrm{ab}$ & $1822.9 \mathrm{a}$ & $2959.9 b$ & $69.50 \mathrm{a}$ & 69.92ab & $71.72 \mathrm{a}$ \\
\hline Oxalic acid & $290.4 \mathrm{c}$ & $1443.4 b$ & $2021.2 d$ & $67.39 b$ & $67.67 \mathrm{~b}$ & $68.32 b$ \\
\hline Salicylic acid & $397.6 \mathrm{a}$ & $2047.8 \mathrm{a}$ & $3263.1 \mathrm{a}$ & $70.18 \mathrm{a}$ & $70.66 a$ & $72.03 \mathrm{a}$ \\
\hline Tocopherol & $351.6 \mathrm{~b}$ & $1891.6 \mathrm{a}$ & $2664.4 \mathrm{c}$ & $69.33 \mathrm{a}$ & $69.54 \mathrm{ab}$ & $71.50 \mathrm{a}$ \\
\hline
\end{tabular}

Values having the same alphabetical letter (s) did not significantly differ at 0.05 level of significance, according to Duncan's multiple range test. 
Table (6): Effect of antioxidant concentrations (ppm) on plant height, number of branches and number of leaves of sweet pepper during 2012 and 2013 seasons.

\begin{tabular}{|c|c|c|c|c|c|c|c|c|c|}
\hline \multirow{3}{*}{ Character } & \multicolumn{3}{|c|}{ Plant height (cm) } & \multicolumn{3}{|c|}{ No. of branches/ plant } & \multicolumn{3}{|c|}{ No. of leaves/plant } \\
\hline & \multicolumn{9}{|c|}{ Days after transplanting } \\
\hline & 30 & 50 & 70 & 30 & 50 & 70 & 30 & 50 & 70 \\
\hline \multicolumn{10}{|c|}{ First season (2012) } \\
\hline 0.0 & $16.97 \mathrm{c}$ & $29.23 \mathrm{c}$ & $36.25 \mathrm{c}$ & $1.65 b$ & $4.07 \mathrm{~d}$ & $8.37 \mathrm{c}$ & $30.24 \mathrm{c}$ & $51.22 \mathrm{c}$ & $78.90 \mathrm{c}$ \\
\hline 200 & $22.74 a$ & $39.20 \mathrm{a}$ & $46.10 \mathrm{a}$ & $2.28 \mathrm{a}$ & $5.77 b$ & $11.29 \mathrm{a}$ & $36.73 a$ & $72.78 \mathrm{a}$ & $98.88 \mathrm{a}$ \\
\hline 400 & $22.68 \mathrm{a}$ & $39.86 a$ & $46.05 \mathrm{a}$ & $2.34 \mathrm{a}$ & $6.16 \mathrm{a}$ & $11.11 \mathrm{a}$ & $37.45 \mathrm{a}$ & $74.04 \mathrm{a}$ & $102.55 \mathrm{a}$ \\
\hline 600 & $20.46 b$ & $34.89 b$ & $40.36 b$ & $1.99 \mathrm{ab}$ & $5.00 \mathrm{c}$ & $9.92 b$ & $33.97 \mathrm{~b}$ & $68.16 b$ & $88.59 b$ \\
\hline \multicolumn{10}{|c|}{ Second season (2013) } \\
\hline 0.0 & $17.34 \mathrm{c}$ & $30.05 \mathrm{c}$ & $36.92 \mathrm{c}$ & $1.62 b$ & $4.25 \mathrm{c}$ & $8.33 c$ & $30.70 \mathrm{c}$ & $52.86 \mathrm{c}$ & $81.55 \mathrm{c}$ \\
\hline 200 & $23.13 \mathrm{a}$ & $40.15 a$ & $47.18 \mathrm{a}$ & $2.46 \mathrm{a}$ & $5.99 a$ & $11.58 \mathrm{a}$ & $38.43 \mathrm{a}$ & $77.56 a$ & $106.38 \mathrm{a}$ \\
\hline 400 & $23.31 \mathrm{a}$ & $40.90 \mathrm{a}$ & $47.49 a$ & $2.58 \mathrm{a}$ & $6.30 \mathrm{a}$ & $11.33 \mathrm{a}$ & $39.31 \mathrm{a}$ & $79.44 a$ & $108.45 \mathrm{a}$ \\
\hline 600 & $21.38 \mathrm{~b}$ & $35.91 \mathrm{~b}$ & $42.44 b$ & $2.20 \mathrm{a}$ & $5.15 b$ & $10.03 b$ & $34.29 \mathrm{~b}$ & $72.45 b$ & $91.72 b$ \\
\hline
\end{tabular}

Values having the same alphabetical letter (s) did not significantly differ at 0.05 level of significance, according to Duncan's s multiple range test.

\section{Fresh and dry weights of plant}

It is obvious from the data presented in Table 7 that spraying with $400 \mathrm{ppm}$ and 200 ppm from any antioxidant had the highest significant values of fresh and dry weights for branches and leaves of sweet pepper in all dates in the first and second seasons.

\section{Leaf area and total chlorophyll}

Data in Table 8 show significant effects for antioxidants on leaf area and total chlorophyll of sweet pepper plant. It could be observed from the data that leaf area per pepper plant and total chlorophyll at all sampling dates had the highest values with application of 200 and $400 \mathrm{ppm}$ of any antioxidant in both seasons. At 50 and 70 days from transplanting spraying at 400 ppm had the best value for leaf area and total chlorophyll in both seasons.
Effect of interaction between antioxidants and their concentrations

Plant height and number of both branches and leaves

Data in Table 9 show significant effects for the interaction between foliar spray of antioxidants and their concentrations on plant height, number of branches per plants as well as number of leaves per plant of sweet pepper. The highest values were recorded with application of $400 \mathrm{ppm}$ followed by $200 \mathrm{ppm}$ of Salicylic acid or $400 \mathrm{ppm}$ of ascorbic acid in both seasons at all dates.

\section{Plant fresh and dry weights}

The effects of the interaction between antioxidants and their concentrations on the fresh and dry weights; viz, branches and leaves of pepper plants are presented in Table 10. All measured parameters gave highly 
SINAI Journal of Applied Sciences (ISSN: 2314-6079) Vol. (6) Is. (1), Apr., 2017

Table (7): Effect of antioxidant concentrations (ppm) on fresh and dry weight ${ }^{\mathrm{s}}$ of both branches and leaves of sweet pepper during 2012 and 2013 seasons.

\begin{tabular}{|c|c|c|c|c|c|c|c|c|c|c|c|c|}
\hline \multirow{4}{*}{ Character } & \multicolumn{6}{|c|}{ Fresh weight (g) } & \multicolumn{6}{|c|}{ Dry weight (g) } \\
\hline & \multicolumn{3}{|c|}{ Branches } & \multicolumn{3}{|c|}{ Leaves } & \multicolumn{3}{|c|}{ Branches } & \multicolumn{3}{|c|}{ Leaves } \\
\hline & \multicolumn{12}{|c|}{ Days after transplanting } \\
\hline & 30 & 50 & 70 & 30 & 50 & 70 & 30 & $\mathbf{5 0}$ & 70 & 30 & 50 & 70 \\
\hline & \multicolumn{12}{|c|}{ First season (2012) } \\
\hline 0.0 & $3.63 d$ & $18.26 \mathrm{~d}$ & $36.19 \mathrm{c}$ & $8.39 c$ & $30.56 \mathrm{c}$ & $51.10 \mathrm{c}$ & $1.12 \mathrm{c}$ & $3.46 \mathrm{c}$ & $8.82 \mathrm{~d}$ & $1.32 \mathrm{c}$ & $4.03 \mathrm{~d}$ & $9.51 \mathrm{~d}$ \\
\hline 200 & $6.68 b$ & $25.26 b$ & $63.56 \mathrm{a}$ & $10.91 \mathrm{a}$ & $46.53 a$ & $75.68 \mathrm{a}$ & $2.09 \mathrm{a}$ & $6.40 \mathrm{a}$ & $13.15 b$ & $2.58 \mathrm{a}$ & $6.63 b$ & $13.34 b$ \\
\hline 400 & $7.36 \mathrm{a}$ & $26.91 \mathrm{a}$ & $63.03 \mathrm{a}$ & $11.25 \mathrm{a}$ & $47.12 \mathrm{a}$ & $77.01 \mathrm{a}$ & $2.15 \mathrm{a}$ & $6.56 \mathrm{a}$ & $13.45 \mathrm{a}$ & $2.55 \mathrm{a}$ & $7.21 \mathrm{a}$ & $14.03 \mathrm{a}$ \\
\hline 600 & $5.91 \mathrm{c}$ & $22.85 \mathrm{c}$ & $55.87 \mathrm{~b}$ & $9.92 b$ & $40.35 b$ & $69.42 b$ & $1.66 \mathrm{~b}$ & $4.95 b$ & $11.78 \mathrm{c}$ & $1.81 \mathrm{~b}$ & $5.40 \mathrm{c}$ & $11.74 \mathrm{c}$ \\
\hline \multicolumn{13}{|c|}{ Second season (2013) } \\
\hline 0.0 & $3.69 \mathrm{c}$ & $18.38 \mathrm{~d}$ & $36.75 \mathrm{c}$ & $8.53 c$ & $30.65 \mathrm{c}$ & $50.95 \mathrm{c}$ & $1.18 \mathrm{c}$ & $3.47 \mathrm{~d}$ & $9.03 \mathrm{~d}$ & $1.36 \mathrm{c}$ & $4.08 \mathrm{~d}$ & $9.63 d$ \\
\hline 200 & $7.23 \mathrm{a}$ & $25.79 b$ & $64.57 \mathrm{a}$ & $11.51 \mathrm{a}$ & $47.77 \mathrm{a}$ & $77.11 \mathrm{a}$ & $2.19 a$ & $6.45 b$ & $13.36 b$ & $2.65 \mathrm{a}$ & $7.02 b$ & $13.84 b$ \\
\hline 400 & $7.53 \mathrm{a}$ & $27.35 \mathrm{a}$ & $63.80 \mathrm{a}$ & $11.70 \mathrm{a}$ & $48.15 \mathrm{a}$ & $78.89 a$ & $2.24 \mathrm{a}$ & $6.61 \mathrm{a}$ & $13.59 \mathrm{a}$ & $2.68 \mathrm{a}$ & $7.31 \mathrm{a}$ & $14.70 \mathrm{a}$ \\
\hline 600 & $5.92 b$ & $23.18 \mathrm{c}$ & $56.74 b$ & $10.42 b$ & $41.18 b$ & $71.04 b$ & $1.74 b$ & $5.01 \mathrm{c}$ & $11.97 \mathrm{c}$ & $1.95 \mathrm{~b}$ & $5.68 \mathrm{c}$ & $11.84 \mathrm{c}$ \\
\hline
\end{tabular}

Table (8): Effect of antioxidant concentrations (ppm) on leaf area and total chlorophyll reading (SPAD) of sweet pepper during 2012 and 2013 seasons.

\begin{tabular}{|c|c|c|c|c|c|c|}
\hline \multirow{3}{*}{ Treatment Character } & \multicolumn{3}{|c|}{ Leaf are /plant $\left(\mathrm{cm}^{2}\right)$} & \multicolumn{3}{|c|}{ Total Chlorophyll Reading (SPAD) } \\
\hline & \multicolumn{6}{|c|}{ Days after transplanting } \\
\hline & 30 & 50 & 70 & 30 & 50 & 70 \\
\hline & \multicolumn{6}{|c|}{ First season (2012) } \\
\hline 0.0 & $231.1 \mathrm{c}$ & $926.1 \mathrm{~d}$ & $1854.0 \mathrm{c}$ & $65.80 \mathrm{c}$ & $65.75 b$ & $66.63 \mathrm{c}$ \\
\hline 200 & $367.8 \mathrm{a}$ & $1797.2 b$ & $3346.5 \mathrm{a}$ & $71.44 a$ & $71.06 \mathrm{a}$ & $72.01 \mathrm{a}$ \\
\hline 400 & $394.4 a$ & 1933.3a & $3619.3 a$ & 71.27ab & $71.98 \mathrm{a}$ & $73.05 \mathrm{a}$ \\
\hline 600 & $316.0 \mathrm{~b}$ & $1637.3 \mathrm{c}$ & $2794.7 b$ & $69.20 \mathrm{~b}$ & $70.26 \mathrm{a}$ & $70.30 \mathrm{~b}$ \\
\hline \multicolumn{7}{|c|}{ Second season (2013) } \\
\hline 0.0 & $237.5 d$ & $985.9 \mathrm{c}$ & $1784.8 \mathrm{~d}$ & $65.40 \mathrm{c}$ & $65.76 b$ & $66.86 b$ \\
\hline 200 & $388.9 \mathrm{~b}$ & $2127.6 \mathrm{a}$ & $3045.1 \mathrm{~b}$ & $70.67 \mathrm{ab}$ & $71.04 \mathrm{a}$ & $72.69 \mathrm{a}$ \\
\hline 400 & $429.8 \mathrm{a}$ & $2224.7 \mathrm{a}$ & $3458.4 \mathrm{a}$ & $71.08 \mathrm{a}$ & $71.27 \mathrm{a}$ & $73.00 \mathrm{a}$ \\
\hline 600 & $342.8 \mathrm{c}$ & $1867.4 \mathrm{~b}$ & $2620.3 \mathrm{c}$ & $69.24 b$ & $69.73 a$ & $71.03 \mathrm{a}$ \\
\hline
\end{tabular}


Table (9): Effect of interaction between some antioxidants and their concentrations (ppm) on plant height, number of branches and number of leaves of sweet pepper during 2012 and 2013 seasons.

\begin{tabular}{|c|c|c|c|c|c|c|c|c|c|c|}
\hline \multirow{3}{*}{$\begin{array}{l}\text { Char } \\
\text { Treatment }\end{array}$} & \multirow[t]{3}{*}{ ter } & \multicolumn{6}{|c|}{ No. of branches/ plant } & \multicolumn{3}{|c|}{ No. of leaves/plant } \\
\hline & & \multicolumn{9}{|c|}{ Days after transplanting } \\
\hline & & 30 & 50 & 70 & 30 & 50 & 70 & 30 & 50 & 70 \\
\hline & & \multicolumn{9}{|c|}{ First season (2012) } \\
\hline \multirow{4}{*}{$\begin{array}{c}\text { Ascorbic } \\
\text { acid }\end{array}$} & 0.0 & $17.8 \mathrm{gh}$ & $29.03 h$ & $36.9 \mathrm{gh}$ & $1.73 \mathrm{ef}$ & $4.15 \mathrm{f}$ & $8.49 \mathrm{~h}$ & $30.27 \mathrm{~g}$ & $51.59 \mathrm{i}$ & $80.93 \mathrm{~g}-\mathrm{i}$ \\
\hline & 200 & $22.52 \mathrm{~d}$ & $40.39 \mathrm{c}$ & $47.40 \mathrm{c}$ & $2.3 b-d$ & $5.66 \mathrm{~cd}$ & $11.53 \mathrm{c}$ & $37.92 b$ & $73.78 \mathrm{c}-\mathrm{e}$ & $102.98 \mathrm{~cd}$ \\
\hline & 400 & $23.74 \mathrm{c}$ & $42.02 b$ & $49.43 b$ & $2.70 \mathrm{ab}$ & $6.64 b$ & $12.20 \mathrm{~b}$ & $40.87 \mathrm{a}$ & $77.42 b$ & $113.97 \mathrm{ab}$ \\
\hline & 600 & $20.65 \mathrm{e}$ & $36.45 \mathrm{e}$ & 41.90ef & $1.91 \mathrm{~d}-\mathrm{f}$ & $4.86 \mathrm{e}$ & $10.31 \mathrm{e}$ & $34.70 \mathrm{c}$ & $68.11 \mathrm{fg}$ & $95.16 \mathrm{~d}-\mathrm{f}$ \\
\hline \multirow{4}{*}{ Oxalic acid } & 0.0 & $17.11 \mathrm{hi}$ & $29.17 \mathrm{~h}$ & $35.85 \mathrm{~h}$ & $1.58 \mathrm{f}$ & $3.91 \mathrm{f}$ & $8.54 \mathrm{~h}$ & $29.77 \mathrm{~g}$ & $51.00 \mathrm{i}$ & $76.56 \mathrm{i}$ \\
\hline & 200 & $20.86 \mathrm{e}$ & $33.46 \mathrm{f}$ & $40.66 f$ & $2.16 c-e$ & $5.01 \mathrm{e}$ & 10.6de & $34.96 \mathrm{c}$ & $68.22 \mathrm{fg}$ & $88.42 \mathrm{fg}$ \\
\hline & 400 & $19.53 \mathrm{f}$ & $31.31 \mathrm{~g}$ & $37.83 \mathrm{~g}$ & $1.76 \mathrm{ef}$ & $4.67 \mathrm{e}$ & $9.64 f$ & 32.96de & $66.63 \mathrm{gh}$ & $86.00 \mathrm{f}-\mathrm{h}$ \\
\hline & 600 & $18.40 \mathrm{~g}$ & $30.32 \mathrm{gh}$ & $36.9 \mathrm{gh}$ & $1.66 \mathrm{f}$ & $4.22 \mathrm{f}$ & $9.26 \mathrm{fg}$ & $32.19 \mathrm{ef}$ & $64.71 \mathrm{~h}$ & 78.29hi \\
\hline \multirow{4}{*}{$\begin{array}{c}\text { Salicylic } \\
\text { acid }\end{array}$} & 0.0 & $16.22 \mathrm{i}$ & $29.47 \mathrm{~h}$ & $36.18 \mathrm{~h}$ & $1.70 \mathrm{f}$ & $4.08 \mathrm{f}$ & $8.18 \mathrm{~h}$ & $31.07 \mathrm{fg}$ & $51.86 \mathrm{i}$ & 79.16hi \\
\hline & 200 & $24.90 \mathrm{~b}$ & $43.00 \mathrm{~b}$ & $50.73 b$ & $2.53 \mathrm{a}-\mathrm{c}$ & $6.00 \mathrm{c}$ & $12.15 b$ & $38.48 b$ & $75.01 b c$ & $106.55 b c$ \\
\hline & 400 & $26.71 \mathrm{a}$ & $47.35 \mathrm{a}$ & $54.00 \mathrm{a}$ & $2.93 \mathrm{a}$ & $7.56 \mathrm{a}$ & $13.18 \mathrm{a}$ & $41.07 \mathrm{a}$ & $81.54 a$ & $116.76 a$ \\
\hline & 600 & $22.63 \mathrm{~d}$ & $36.96 \mathrm{e}$ & $41.56 \mathrm{ef}$ & $2.46 b c$ & $5.51 d$ & $10.99 \mathrm{~d}$ & $34.65 \mathrm{c}$ & $71.06 \mathrm{~d}-\mathrm{f}$ & $94.22 \mathrm{~d}-\mathrm{f}$ \\
\hline \multirow{5}{*}{ Tocopherol } & 0.0 & 17.09hi & $29.27 \mathrm{~h}$ & $36.06 \mathrm{~h}$ & $1.58 \mathrm{f}$ & $4.11 \mathrm{f}$ & $8.28 \mathrm{~h}$ & $29.84 \mathrm{~g}$ & $50.45 \mathrm{i}$ & 78.96hi \\
\hline & 200 & $22.67 d$ & $39.95 \mathrm{~cd}$ & $45.62 d$ & $2.16 \mathrm{c}-\mathrm{e}$ & $6.41 b$ & $10.86 \mathrm{~d}$ & $35.56 \mathrm{c}$ & $74.11 \mathrm{~cd}$ & $97.55 \mathrm{de}$ \\
\hline & 400 & $20.75 \mathrm{e}$ & $38.77 \mathrm{~d}$ & $42.94 \mathrm{e}$ & $1.96 \mathrm{~d}-\mathrm{f}$ & $5.75 \mathrm{~cd}$ & $9.44 \mathrm{fg}$ & $34.89 \mathrm{c}$ & 70.59ef & $93.50 \mathrm{ef}$ \\
\hline & 600 & $20.17 \mathrm{ef}$ & $35.84 \mathrm{e}$ & $41.14 \mathrm{f}$ & $1.91 \mathrm{~d}-\mathrm{f}$ & $5.42 d$ & $9.15 \mathrm{~g}$ & $34.37 \mathrm{~cd}$ & $68.77 \mathrm{fg}$ & $86.68 f-h$ \\
\hline & \multicolumn{10}{|c|}{ Second season( 2013) } \\
\hline \multirow{4}{*}{$\begin{array}{c}\text { Ascorbic } \\
\text { acid }\end{array}$} & 0.0 & $17.58 \mathrm{i}$ & $29.39 \mathrm{i}$ & $37.3 \mathrm{fg}$ & $1.75 \mathrm{~g}-\mathrm{i}$ & $4.42 \mathrm{gh}$ & $8.62 \mathrm{i}$ & $31.12 \mathrm{~g}$ & $52.97 \mathrm{~g}$ & $83.00 \mathrm{~h}$ \\
\hline & 200 & $23.22 \mathrm{~d}$ & $41.06 \mathrm{c}$ & $48.11 \mathrm{c}$ & $2.66 b-d$ & $5.98 \mathrm{de}$ & $11.73 \mathrm{c}$ & $39.44 \mathrm{c}$ & $77.60 \mathrm{~cd}$ & $111.62 \mathrm{c}$ \\
\hline & 400 & $24.3 \mathrm{bc}$ & $43.93 b$ & $51.61 \mathrm{~b}$ & $2.90 \mathrm{ab}$ & $6.93 b$ & $12.59 \mathrm{~b}$ & $42.87 b$ & $81.98 b$ & $120.26 b$ \\
\hline & 600 & $21.94 \mathrm{e}$ & $37.00 \mathrm{e}$ & $44.68 \mathrm{~d}$ & $2.58 b-d$ & $5.01 \mathrm{f}$ & $10.57 \mathrm{e}$ & $35.16 \mathrm{ef}$ & $71.01 \mathrm{e}$ & $97.03 \mathrm{ef}$ \\
\hline \multirow{4}{*}{ Oxalic acid } & 0.0 & $17.04 \mathrm{i}$ & 30.06hi & $36.53 \mathrm{~g}$ & $1.40 \mathrm{i}$ & $4.33 \mathrm{~h}$ & $8.13 \mathrm{j}$ & $30.02 \mathrm{~g}$ & $51.99 \mathrm{~g}$ & $80.63 \mathrm{~h}$ \\
\hline & 200 & $20.73 \mathrm{~g}$ & $34.18 \mathrm{f}$ & $41.74 \mathrm{e}$ & $2.26 \mathrm{c}-\mathrm{f}$ & $5.15 f$ & $10.68 \mathrm{e}$ & $36.30 \mathrm{de}$ & $73.73 \mathrm{de}$ & $91.26 \mathrm{fg}$ \\
\hline & 400 & $19.9 \mathrm{gh}$ & $32.51 \mathrm{~g}$ & $38.50 \mathrm{f}$ & $2.13 \mathrm{e}-\mathrm{g}$ & $4.75 \mathrm{fg}$ & $9.69 \mathrm{fg}$ & $33.40 \mathrm{f}$ & $69.86 \mathrm{e}$ & $85.50 \mathrm{gh}$ \\
\hline & 600 & $19.36 \mathrm{~h}$ & $31.92 \mathrm{~g}$ & $36.96 \mathrm{~g}$ & $1.91 \mathrm{f}-\mathrm{h}$ & $4.14 \mathrm{~h}$ & $9.17 \mathrm{~h}$ & $31.30 \mathrm{~g}$ & $65.66 f$ & $82.10 \mathrm{~h}$ \\
\hline \multirow{4}{*}{$\begin{array}{c}\text { Salicylic } \\
\text { acid }\end{array}$} & 0.0 & $16.91 \mathrm{i}$ & $30.51 \mathrm{~h}$ & $36.86 \mathrm{~g}$ & $1.70 \mathrm{~g}-\mathrm{i}$ & $4.11 \mathrm{~h}$ & $8.33 \mathrm{ij}$ & $30.97 \mathrm{~g}$ & $52.94 \mathrm{~g}$ & $81.56 \mathrm{~h}$ \\
\hline & 200 & $25.27 b$ & $43.65 b$ & $51.81 \mathrm{~b}$ & $2.70 \mathrm{bc}$ & $6.23 \mathrm{~cd}$ & $12.58 \mathrm{~b}$ & $40.57 \mathrm{c}$ & $81.26 \mathrm{bc}$ & $117.3 \mathrm{bc}$ \\
\hline & 400 & $27.09 \mathrm{a}$ & $48.02 \mathrm{a}$ & $55.20 \mathrm{a}$ & $3.23 \mathrm{a}$ & $7.63 a$ & $13.33 \mathrm{a}$ & $45.03 a$ & $90.64 a$ & $130.03 a$ \\
\hline & 600 & $23.4 \mathrm{~cd}$ & $38.65 d$ & $45.36 \mathrm{~d}$ & $2.40 \mathrm{c}-\mathrm{e}$ & $5.81 \mathrm{de}$ & $11.11 \mathrm{~d}$ & $35.49 \mathrm{de}$ & $77.93 \mathrm{~cd}$ & $97.46 \mathrm{ef}$ \\
\hline \multirow{4}{*}{ Tocopherol } & 0.0 & $17.81 \mathrm{i}$ & $30.25 \mathrm{hi}$ & $36.90 \mathrm{~g}$ & $1.66 \mathrm{hi}$ & $4.16 \mathrm{~h}$ & $8.23 \mathrm{ij}$ & $30.68 \mathrm{~g}$ & $53.55 \mathrm{~g}$ & $81.00 \mathrm{~h}$ \\
\hline & 200 & $23.3 \mathrm{~cd}$ & $41.71 \mathrm{c}$ & $47.07 \mathrm{c}$ & $2.23 \mathrm{~d}-\mathrm{f}$ & $6.61 b c$ & $11.3 \mathrm{~cd}$ & $37.41 \mathrm{~d}$ & $77.65 \mathrm{~cd}$ & $105.32 \mathrm{~d}$ \\
\hline & 400 & $21.8 \mathrm{ef}$ & $39.13 d$ & $44.67 \mathrm{~d}$ & $2.06 \mathrm{e}-\mathrm{h}$ & $5.90 \mathrm{de}$ & $9.73 \mathrm{f}$ & $35.96 \mathrm{de}$ & $75.30 \mathrm{~d}$ & $98.01 \mathrm{e}$ \\
\hline & 600 & $20.8 \mathrm{fg}$ & $36.08 \mathrm{e}$ & $42.79 \mathrm{e}$ & $1.90 \mathrm{f}-\mathrm{h}$ & $5.61 \mathrm{e}$ & $9.29 \mathrm{gh}$ & $35.21 \mathrm{ef}$ & $75.20 \mathrm{~d}$ & $90.30 \mathrm{~g}$ \\
\hline
\end{tabular}

Values having the same alphabetical letter (s) did not significantly differ at 0.05 level of significance, according to Duncan's multiple range test. 
SINAI Journal of Applied Sciences (ISSN: 2314-6079) Vol. (6) Is. (1), Apr. 2017

Table (10): Effect of interaction between antioxidants and their concentrations (ppm) on fresh and dry weight $t^{s}$ of both branches and leaves of sweet pepper during 2012 and 2013 seasons

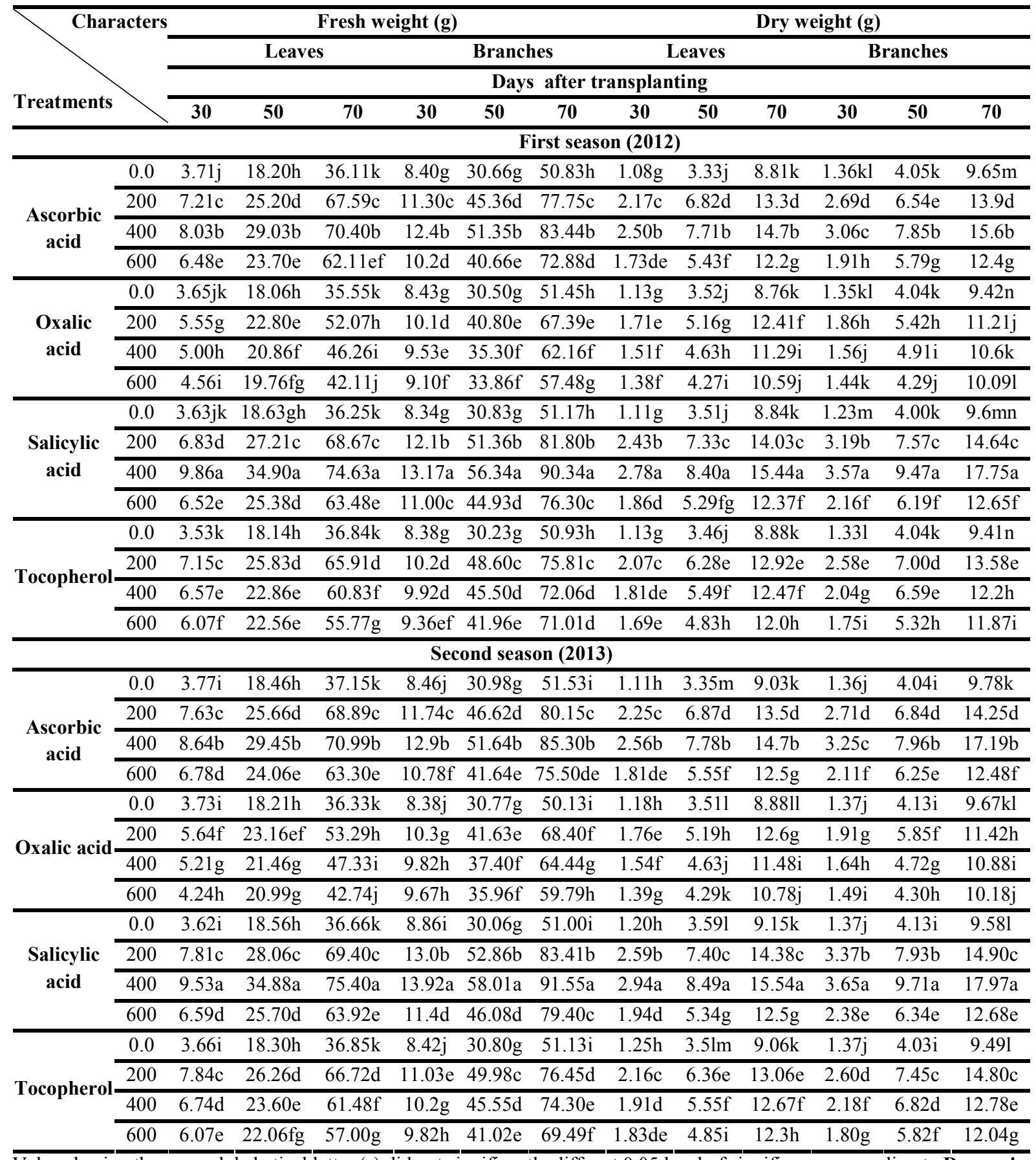

Values having the same alphabetical letter (s) did not significantly differ at 0.05 level of significance, according to Duncan's multiple range test. 
significant differences among the treatments at all dates. Application of $400 \mathrm{ppm}$ from salicylic acid had the highest values followed by ascorbic acid with $400 \mathrm{ppm}$ at 30,50 , and 70 days from transplanting in both growing seasons.

\section{Leaf area and total chlorophyll}

Leaf area and total chlorophyll of sweet pepper at all dates in both growing seasons were significantly affected by the interaction between antioxidants and their concentrations Table 11. The interaction between salicylic acid and $400 \mathrm{ppm}$ at all dates recorded the highest value for leaf area in both growing seasons. Interaction between ascorbic acid with $400 \mathrm{ppm}$, salicylic acid with 200 and $400 \mathrm{ppm}$ and tocopherol with $200 \mathrm{ppm}$ had the best value for total chlorophyll in both seasons.

It could be concluded that, the increase in vegetative growth characters caused by antioxidants may be due to the role of antioxidants intercept free radicals and protect cells from the oxidative damage that leads to aging and disease (Wada and Ou, 2002; Karadeniz et al., 2005). Antioxidants play a role in the reduction or prevention of enzymatic reactions by inhibiting polyphenol oxidase (Maurice et al., 2000).

Salicylic Acid (SA) is a growth regulator which participates in the regulation for physiological and biochemical processes in plants (Bhupinder and Usha, 2003). It is an endogenous hormone which stimulates plant growth and is associated with increased amount of water content in the plant cell (Hayat et al., 2010). Foliar application of $\mathrm{SA}$ is also involved in stomata regulation thereby can work the controlling to photosynthetic rate, consequently, enhanced photosynthesis, also it increased sap production in the leaf lamella which resulted in maintenance of relative water content in leaf and better growth (Khan et al., 2003; Hayat et al., 2010). Pacheco et al. (2013) suggested that the observed increase in photosynthesis rate in plants sprayed with SA can be assigned to metabolic changes at the chloroplasts level (efficiency of photosystem II and Rubisco enzyme activity).

These results are in agreement with those obtained by Fathy et al. (2000) on eggplant who mentioned that salicylic acid increased plant height, number of branches and leaves per plant and plant dry weight. Previous studies have demonstrated that wide range of responses might appear after exogenous SA application as follows: increases in tomato plant height, number of flower branches and number of leaves (Ali et al., 2009).

Yildirim and Dursun, (2009) on tomato mentioned that lower SA concentration increased plant height, number of branches, leaves per plant and dry weight. Also, many researches come to similar results (Akbarimehr et al., 2013 on sweet pepper; Kazemi, 2014 on tomato; Abdul Qados, Amira, 2015 on sweet pepper and Abd ElGawad and Bondok, 5015) on tomato.

The positive effects of SA could be attributed to the increase of $\mathrm{Co}_{2}$ assimilation and photosynthetic rate, and increase of mineral uptake by stressed plant under SA treatment (Khan et al., 2003; Szepesi et al., 2005). Some researches indicated that salicylic acid increases membrane permeability and that would facilitates absorption and utilization of mineral nutrients and transport of assimilates (Javaheri et al., 2012).

Salicylic acid acts as one of antioxidant substances concentreated in the chloroplast and protect the photosynthetic apparatus when a plant is subjected to stress, by scavenging the excessively free redicals (ROS) (Sreenivasulu et al., 2000). These results are in agreement with those obtained by Canakci (2011) who found that $1.5 \mathrm{mM}$ concentration of salicylic acid had a stimulating effect on growth, dry weight and protein of pepper as compared with other concentrations ( 5 and $10 \mathrm{mM}$ ). 
SINAI Journal of Applied Sciences (ISSN: 2314-6079) Vol. (6) Is. (1), Apr. 2017

Table (11): Effect of interaction between antioxidants and their concentrations (ppm) on leaf area and total chlorophyll reading (SPAD) of sweet pepper during 2012 and 2013 seasons.

\begin{tabular}{|c|c|c|c|c|c|c|c|}
\hline \multirow{3}{*}{ Treatments } & \multirow[t]{2}{*}{ Characters } & & \multicolumn{2}{|c|}{ Leaf area/ plant $\left(\mathrm{cm}^{2}\right)$} & \multicolumn{3}{|c|}{ Total Chlorophyll Reading (S } \\
\hline & & & \multicolumn{5}{|c|}{ Days after transplanting } \\
\hline & & 30 & 50 & 70 & 30 & 50 & 70 \\
\hline & & & \multicolumn{5}{|c|}{ First season (2012) } \\
\hline \multirow{4}{*}{$\begin{array}{c}\text { Ascorbic } \\
\text { Acid }\end{array}$} & 0.0 & $233.5 \mathrm{j}$ & $924.2 \mathrm{~h}$ & $1852.6 f$ & $65.53 \mathrm{~g}$ & $66.83 \mathrm{de}$ & $66.76 \mathrm{gh}$ \\
\hline & 200 & $372.7 \mathrm{~cd}$ & $1810.9 \mathrm{~cd}$ & $3602.0 \mathrm{c}$ & $70.56 b-d$ & $72.00 \mathrm{bc}$ & $72.63 \mathrm{~cd}$ \\
\hline & 400 & $441.5 b$ & $2102.5 b$ & $4101.4 b$ & $72.43 a-c$ & $74.20 \mathrm{ab}$ & $75.03 b$ \\
\hline & 600 & $304.5 \mathrm{gh}$ & $1689.5 \mathrm{de}$ & $3015.1 d$ & $69.53 \mathrm{de}$ & $71.10 \mathrm{c}$ & $71.43 \mathrm{de}$ \\
\hline \multirow{4}{*}{ Oxalic acid } & 0.0 & $231.1 \mathrm{j}$ & $931.9 \mathrm{~h}$ & $1827.5 f$ & $65.80 \mathrm{~g}$ & $65.00 \mathrm{de}$ & $66.46 \mathrm{~h}$ \\
\hline & 200 & $331.59 \mathrm{fg}$ & $1488.9 \mathrm{fg}$ & $2433.9 \mathrm{e}$ & $69.73 \mathrm{de}$ & $67.40 \mathrm{~d}$ & $69.16 f$ \\
\hline & 400 & $281.3 \mathrm{hi}$ & $1403.1 \mathrm{~g}$ & $2145.9 \mathrm{ef}$ & $68.10 \mathrm{ef}$ & $67.23 \mathrm{de}$ & $68.26 \mathrm{fg}$ \\
\hline & 600 & $264.2 \mathrm{i}$ & $1484.8 \mathrm{fg}$ & $1998.7 f$ & $68.26 \mathrm{~d}-\mathrm{f}$ & $65.95 \mathrm{de}$ & $66.93 \mathrm{gh}$ \\
\hline \multirow{4}{*}{$\begin{array}{c}\text { Salicylic } \\
\text { Acid }\end{array}$} & 0.0 & $230.5 \mathrm{j}$ & $933.4 \mathrm{~h}$ & $1866.4 f$ & $65.20 \mathrm{~g}$ & $66.83 \mathrm{de}$ & $66.40 \mathrm{~h}$ \\
\hline & 200 & $400.2 c$ & $2036.8 b$ & $4025.6 b$ & $72.70 \mathrm{ab}$ & $73.80 a-c$ & $73.93 b c$ \\
\hline & 400 & $514.8 \mathrm{a}$ & $2529.2 \mathrm{a}$ & $5086.8 \mathrm{a}$ & $74.33 \mathrm{a}$ & $75.23 a$ & $76.93 a$ \\
\hline & 600 & $339.6 \mathrm{ef}$ & $1790.6 \mathrm{~cd}$ & $3089.6 \mathrm{~d}$ & 70.06de & $72.56 a-c$ & 71.96de \\
\hline \multirow{4}{*}{ Tocopherol } & 0.0 & $229.2 j$ & $914.7 \mathrm{~h}$ & $1869.6 \mathrm{f}$ & $66.70 \mathrm{fg}$ & $64.36 \mathrm{e}$ & $66.90 \mathrm{gh}$ \\
\hline & 200 & $366.8 \mathrm{de}$ & $1852.2 \mathrm{c}$ & $3324.4 d$ & $72.76 \mathrm{ab}$ & $71.06 \mathrm{c}$ & $72.33 \mathrm{de}$ \\
\hline & 400 & $339.9 \mathrm{ef}$ & $1698.3 \mathrm{de}$ & $3143.1 d$ & $70.23 c-e$ & $71.29 b c$ & 71.96de \\
\hline & 600 & $355.6 \mathrm{def}$ & $1584.3 \mathrm{ef}$ & $3075.5 d$ & $68.96 \mathrm{~d}-\mathrm{f}$ & $71.43 b c$ & $70.90 \mathrm{e}$ \\
\hline & & & \multicolumn{5}{|c|}{ Second season (2013) } \\
\hline \multirow{4}{*}{$\begin{array}{c}\text { Ascorbic } \\
\text { Acid }\end{array}$} & 0.0 & $236.4 \mathrm{~h}$ & $955.7 \mathrm{~g}$ & $1834.4 \mathrm{f}$ & $65.43 \mathrm{~g}$ & $66.13 \mathrm{~h}-\mathrm{j}$ & $67.86 \mathrm{~d}-\mathrm{g}$ \\
\hline & 200 & $371.5 \mathrm{e}$ & $2064.9 d$ & $3143.0 \mathrm{~d}$ & $70.40 \mathrm{~cd}$ & $71.13 \mathrm{~cd}$ & $72.56 b c$ \\
\hline & 400 & $471.8 b$ & $2363.2 b$ & $3975.3 b$ & $72.45 \mathrm{a}$ & $72.33 \mathrm{bc}$ & $75.10 \mathrm{a}$ \\
\hline & 600 & $357.8 \mathrm{e}$ & $1907.8 \mathrm{e}$ & $2886.6 \mathrm{~d}$ & $69.72 \mathrm{de}$ & $70.10 \mathrm{~d}$ & $71.36 \mathrm{c}$ \\
\hline \multirow{4}{*}{ Oxalic acid } & 0.0 & $230.5 \mathrm{~h}$ & $973.3 \mathrm{~g}$ & $1828.8 f$ & $65.73 \mathrm{~g}$ & $66.60 \mathrm{~g}-\mathrm{h}$ & $66.53 \mathrm{fg}$ \\
\hline & 200 & $352.8 \mathrm{e}$ & $1796.7 \mathrm{e}$ & $2317.5 \mathrm{e}$ & $68.43 \mathrm{ef}$ & $67.66 f-h$ & $69.40 \mathrm{~d}$ \\
\hline & 400 & $305.7 \mathrm{f}$ & $1545.6 \mathrm{f}$ & $2115.4 \mathrm{e}$ & $68.20 \mathrm{ef}$ & $68.36 \mathrm{ef}$ & $68.33 \mathrm{~d}-\mathrm{f}$ \\
\hline & 600 & $272.6 \mathrm{~g}$ & $1457.7 f$ & $1822.9 f$ & $67.20 \mathrm{f}$ & $68.06 \mathrm{fg}$ & $69.02 \mathrm{de}$ \\
\hline \multirow{4}{*}{$\begin{array}{c}\text { Salicylic } \\
\text { Acid }\end{array}$} & 0.0 & $242.6 \mathrm{~h}$ & $990.0 \mathrm{~g}$ & $1802.5 \mathrm{f}$ & $64.96 \mathrm{~g}$ & $64.66 \mathrm{j}$ & $65.90 \mathrm{~g}$ \\
\hline & 200 & $429.2 c$ & $2336.3 b$ & $3631.6 \mathrm{c}$ & $71.86 \mathrm{a}-\mathrm{c}$ & $72.90 \mathrm{ab}$ & $74.33 \mathrm{ab}$ \\
\hline & 400 & $558.9 \mathrm{a}$ & $2799.5 a$ & $4729.2 \mathrm{a}$ & $73.23 \mathrm{a}$ & $74.06 \mathrm{a}$ & $76.16 a$ \\
\hline & 600 & $359.6 \mathrm{e}$ & $2065.4 d$ & $2889.1 \mathrm{~d}$ & $70.66 b-d$ & $71.03 \mathrm{~cd}$ & $71.73 \mathrm{c}$ \\
\hline \multirow{4}{*}{ Tocopherol } & 0.0 & $240.6 \mathrm{e}$ & $1024.8 \mathrm{~g}$ & $1673.2 \mathrm{f}$ & $65.46 \mathrm{~g}$ & $65.66 \mathrm{ij}$ & $67.16 \mathrm{e}-\mathrm{g}$ \\
\hline & 200 & $401.8 \mathrm{~d}$ & $2312.5 b d$ & $3088.2 d$ & $72.00 \mathrm{ab}$ & $72.46 b c$ & 74.46ab \\
\hline & 400 & $382.5 \mathrm{de}$ & $2190.6 c$ & $3013.7 \mathrm{~d}$ & $70.46 b-d$ & $70.32 d$ & $72.38 b c$ \\
\hline & 600 & $381.4 \mathrm{de}$ & $2038.6 d$ & $2882.5 d$ & $69.40 \mathrm{de}$ & $69.73 \mathrm{de}$ & $72.00 \mathrm{c}$ \\
\hline
\end{tabular}

Values having the same alphabetical letter (s) did not significantly differ at 0.05 level of significance, according to Duncan's multiple range test. 
In addition, some researchers reported that the highest plant growth and number of braches values were obtained from the ascorbic acid foliar application (Shabana, Abeer et al., 2015 on sweet pepper; ElHifny and El- Sayed, 2011 on sweet pepper and Ghurbat, 2013) on pepper plant. These increases in the above parameters by using ascorbic acid may be due to the fact that ascorbic acid as an antioxidant has an effect as plant growth regulators (Johnson et al., 1999) and its role in activating both cell division and elongation in meristematic tissues, as well as the biosynthesis of organic foods (Nijjar, 1985). The beneficial effect of ascorbic acid (Vitamin. C) on plant height may be attributed to the fact that ascorbic acid (Vitamin. C) is involved in the regulation of shoot and root elongation, cell vacuole, leaf area and cell expansion (Sumalan and Carmen, 2002; El Hariri et al., 2010; Farahat et al., 2013).

The stimulating effect of ascorbic acid on plant growth may be attributed to an increase in availability and uptake of water and essential nutrients through adjusting cell osmotic pressure, and reducing the accumulation of harmful free radicals (ROS) by increasing antioxidants and enzyme activities (Farouk et al., 2011). In addition, they indicated that the positive effect of ascorbic acid on plant growth may be due to its effect on increasing nutrient uptake and increase elements content such as nitrogen, phosphorous, and potassium. Phosphorous and potassium are essential nutrients playing an important role in the biosynthesis and translocation of carbohydrates, and necessary for stimulating cell division, cell turger and forming DNA and RNA (Saeidi-Sar $\boldsymbol{e t} \boldsymbol{a l}$., 2013).

These results are in agreement with those obtained by (Azooz and Al-Fredan, 2009; Ekmekçi and Karaman, 2012) who indicated that, vitamins (such as ascorbic acid) could accelerate cell division and cell enlargement and induce improvement of membrane integrity, which may have contributed in reducing ion leakage, and consequently improving growth. Many studies have reported that vitamins, when used with optimal concentration, exhibited beneficial effect on growth and yield of some crop plants grown under saline conditions (Khan et al., 2011; Ekmekçi and Karaman, 2012). Wassel et al. (2007) assumed that the effect of ascorbic acid on the plant growth might be due to the auxinic action of Ascorbic acid as well as, its improved role in many metabolic and physiological processes and enhancing the synthesis of carbohydrates.

The promoting effect of SA on the leaf area was attributed to its important roles on activating cell division and the biosynthesis of organic foods. In addition, Raskin (1992) mentioned that enhancing effect of SA on the availability and movement of nutrients could result in stimulating different nutrients in the leaves. Gharib (2006) on basil and marjoram and Khan et al. (2003) on corn and soybean reported that application of SA enhanced photosynthesis rate, so that leaf area has been increased. The present results are in agreement with those of Abou El-Yazeid, (2011) on sweet pepper; Abdul Qados (2015) on sweet pepper under salt stress conditions, Shabana et al. (2015) on sweet pepper; and Kazemi (2013) on strawberry.

Exogenous SA alters the activities of antioxidant enzymes and increases plant tolerance to abiotic stress by decreasing generation of ROS. It has been found that SA has different effects on stress adaptation and damage development of plants that depend on plant species, concentration, method and time of SA application (Metwally et al., 2003). Kazemi (2014) on tomato found that using low SA $(0.5 \mathrm{~m}$ molL $^{-1}$ ) concentrations alone significantly increased chlorophyll content. Also, Gharib 
(2006) on basil and Yildirim and Dursun (2009) on tomato mentioned that lower SA concentration increased plant height, chlorophyll content, number of branches, leaves per plant and dry weight.

The increase in total chlorophyll under low levels of salinity recorded in this study is in agreement with the finding of Hussein et al., (2012) on pepper plants and Lui et al., (2007) on Aeluropus littoralis plants who found that salt stress increased $\mathrm{Chl}$ a and $\mathrm{Chl} \mathrm{b}$ contents, but the $\mathrm{Chl} \mathrm{a} / \mathrm{Chl} \mathrm{b}$ ratio declined, which implies the stimulation of $\mathrm{Chl}$ a accepted from $\mathrm{NaCl}$ was smaller than that of Chl b. This increase may be attributed to the thickness of the leaves under salt stress rather than to stimulation of pigment formation.

On the other hand, ascorbic acid can mitigate the adverse effects of stress through increasing the content of IAA and GA3 and decreasing ABA level, which may be involved in protecting the photosynthetic apparatus and consequently increasing the photosynthetic pigments (Saeidi-Sar et al., 2013). Consequently, in ascorbic acid treated plants, high level of carotenoids can synergistically function with ascorbic acid to provide an effective barrier against oxidation under salinity stress. These results reinforce the results obtained by other investigators (Azooz and Al-Fredan, 2009) who concluded that, chlorophyll content of plants treated with vitamins (such as ascorbic acid) was increased due to the protection effect of these vitamins.

Azzedine et al. (2011) reported that, ascorbic acid can detoxify and neutralize the reactive oxygen species by prevention of free radicals activity, leading to increase in chlorophyll content of vitamin- treated plants. They also found that, application of vitamin $\mathrm{C}$ (ascorbic acid) was effective to mitigate the adverse effect of abiotic stress on plant growth due to increased leaf area and improved chlorophyll and carotenoids contents. On the contrary, Pacheco et al.
(2013) observed that the chlorophyll content in SA treated marigold plants did not differ significantly from the control plants.

\section{REFERENCES}

Abd El-Gawad, H.G. and Bondok, A.M. (2015). Response of tomato plants to salicylic acid and chitosan under infection with tomato mosaic virus. Ame.-Eurasian J. Agric. and Environ. Sci., 15 (8): 1520-1529.

Abdul Qados, A.M.S. (2015). Effects of salicylic acid on growth, yield and chemical contents of pepper (Capsicum annuum L.) plants grown under salt stress conditions. Intern. J. Agric. and Crop Sci., 8 (2): 107-113.

Abou El-Yazeid, A. (2011). Effect of foliar application of salicylic acid and chelated zinc on growth and productivity of sweet pepper (Capsicum annuum L.) under autumn planting. Res. J. Agric. and Biol. Sci., 7 (6): 423-433.

Akbarimehr, H.; Delshad, M.; Zamani, Z. and Abdossi, V. (2013). Effect of pre-sowing salicylic acid seed treatment on seed germination and growth of greenhouse sweet pepper plants. Indian J. Sci. and Technol., 6 (1): 3868-3871.

Ali, A.A.; Ali, T.B. and Nour, K.A. (2009). Antioxidants and some natural compounds applications in relation to tomato growth, yield and chemical constituents. Ann. Agric. Sci., Moshtohor, 47 (4): 469-477.

Azooz, M.M. and Al-Fredan, M.A. (2009). The inductive role of vitamin $\mathrm{C}$ and its mode of application on growth, water status, antioxidant enzyme activities and protein patterns of Vicia faba L. cv. Hassawi grown under seawater irrigation. Ame. J. Plant. Physiol., 4: 38-51.

Azzedine, F.; Gherroucha, H. and Baka, M. (2011). Improvement of salt tolerance 
in durum wheat by ascorbic acid application. J. Stress Physiol. Biochem., 7: 27-37.

Bhupinder, S. and Usha, K. (2003). Salicylic acid induced physiological and biochemical changes in wheat seedlings under water stress. Plant Growth Regul., 39: 137-141.

Black, C. and Editor, A. (1965). Methods of Soil Analysis, part 1 and 2 Ame. Soc. Agron. In., pub. Madison, Wisconison, USA.

Borsani, O.; Valpuesta, V. and Botella, M.A. (2001). Evidence for a role of salicylic acid in the oxidative damage generated by nacl and osmotic stress in arabidopsis seedling. Plant Physiol., 126: 1024- 1030.

Canakci, S. (2011). Effects of salicylic acid on growth, biochemica constituents in pepper (Capsicum annuum L.) seedlings. Pak. J. Bio. Sci., 14: 300-304.

DellaPenna, D. (2005). Progress in the dissection and manipulation of vitamin $\mathrm{E}$ synthesis. Trends Plant Sci., 10:574-579.

Demiral, T. and Turkan, I. (2005). Comparative lipid peroxidation antioxidant defense systems and proline content in roots of two rice cultivars in salt tolerance. Environ. and Exp. Bot., 53 (3): 247-257.

Duncan, D.B. (1958). Multiple Range and Multiple F-Test. Biomet., 11:1-42.

Ekmekçi, B.A. and Karaman, M. (2012). Exogenous ascorbic acid increases resistance to salt of (Silybum marianum L.). Afr. J. Biotech., 11: 9932-9940.

El-Hariri, D.M.; Sadak, M.S. and ElBassiony, H.M.S. (2010). Response of flax cultivars to ascorbic acid and ATocopherol under salinity stress conditions. Int. J. Acad. Res., 2 (6): 101-109.

El-Hifny, I.M.M. and El-Sayed, M.A.M. (2011). Response of sweet pepper plant growth and productivity by application of ascorbic acid and biofertilizers under saline condition. Aust. J. Basic. and Appl. Sci., 5(6):1273.

Farahat, M.M.; Azza, M.; Mona, H.M. and Sahar, M.Z. (2013). Salt tolerance in grevillea robusta seedlings via foliar application of ascorbic acid. Middle-East J. Sci. Res. 14 (1): 09-15.

Farouk, S.; Mosa, A.A.; Taha, A.A.; Ibrahim, H.M. and El-Gahmery, A.M. (2011). Protective effect of humic acid and chitosan on radish (Raphanus sativus L. var. sativus) plants subjected to cadmium stress. J. Stress Physiol. Biochem., 7(2): 99-116.

Fathy, E.S.L.; Farid, S. and El-Desouky, S.A. (2000). Induce cold tolerance of outdoor tomatoes during early summer season by using ATP, yeast, other natural and chemical treatments to improve their fruiting and yield. J. Agric. Sci., Mansoura Univ., 25 (1): 377-401.

Gharib, F.A. (2006). Effect of salicylic acid on the growth, metabolic activities and oil content of basil and marjoram. Int. J. Agric. and Biol., 8 (4): 485-492.

Gharib, F.A.E. (2007). Effect of salicylic acid on the growth, metabolic activities and oil content of basil and marjoram. Int. J. Agric. and Biol., 9(2): 294-301.

Ghurbat, H.M. (2013). Effect of seamino and ascorbic acid on growth, yield and fruits quality of pepper (Capsicum annuиm L). Int. J. Pure Appl. Sci. Technol., 17(2): 9-16.

Hayat, Q.; Hayat, S.; Irfan, M. and Ahmad, A. (2010). Effect of exogenous salicylic acid under changing environment: A review. Environ. Exp. Bot., 68: 14-25.

Howard, L.R.; Talcott, S.T.; Brenes, C.H. and Villalon, B. (2000). Changes in phytochemical and antioxidant activity of selected pepper cultivars (Capsicum species) as influenced by maturity. J. Agric. Food Chem., 48: 1713 - 1720. 
Hussein, M.M; El-Faham, S.Y. and Alva, A.K. (2012). Pepper plants growth, yield, photosynthetic pigments, and total phenols as affected by foliar application of potassium under different salinity irrigation water. Agric. Sci., 3: 241-248.

Jackson, M.L. (1967). Soil chemical analysis. Prentice hall of India Pvt. Ltd., New Delhi India, 144-197: 326-338.

Javaheri, M.K; Dadkhah, M.A. and Tavallaee, F.Z. (2012). Effects of salicylic acid on yield and quality characters of tomato fruit (Lycopersicum esculentum Mill.). Int. J. Agric. Crop Sci., 4 (16): 1184-1187.

Johnson, J.R.; Fahy, D.; Gish, N. and Andrews, P.K. (1999). Influence of ascorbic acid sprays on apple sunburn, Good Fruit Grower., 50(13): 81-83.

Karadeniz F.; Burdurlu, H.S.; Koca, N. and Soyer, Y. (2005). Antioxidant activity of selected fruits and vegetables grown in Turkey. Turk. J. Agric., 29: 197-203.

Kazemi, M. (2013). Foliar application of salicylic acid and calcium on yield, yield component and chemical properties of strawberry. Bull. Evn. Pharmacol. Life Sci., 2 (11): 19-23.

Kazemi, M. (2014). Effect of Foliar Application with Salicylic Acid and Methyl Jasmonate on Growth, Flowering, Yield and Fruit Quality of Tomato. Bull. Env. Pharmacol. Life Sci., (3): 154-158.

Khan, T.A.; Mazid, M. and Mohammad, F. (2011). A review of ascorbic acid potentialities against oxidative stress induced in plants. J. Agrobiol., 28 (2): 97-111.

Khan, W.; Prithiviraj, B. and Smith, D.L. (2003). Photosynthetic responses of corn and soybean to foliar application of salicylates. J. Plant Physiol., 160: 485492.
Lui Z.H; Shi, L.R.; Bai, L.R. and Zhao, K.F. (2007). Effects of salt stress on the contents of chlorophyll and organic solutes in Aeluropus littoralis var. sinensis Debeaux. Zhi Wu Sheng Li Yu Fen Zi Sheng Wu Xue Xue Bao., 33 (2): 165-72.

Malencic, D.J.; Vasic, D.; Popovic, M. and Devic, D. (2004). Antioxidant systems in sunflower as affected by oxalic acid. Biologia Plantarum, 48: 243-247.

Mano, J. (2002). Early events in environmental stress. Taylor Francis pub., 217-245.

Maurice, R.M.; Kim, J. and Wei, C.I. (2000). Enzymatic browning in fruits, vegetables and seafoods. Food Science and Human Nutrition Department. University Florida, 65: 791-795.

Metwally, A.; Finkemeier, I.; Georgi, M.; Dietz, K.J. (2003). Salicylic acid alleviates the cadmium toxicity in barley seedlings. Plant Physiol., 132: 272-281.

Navarro, J.M.; Flores, P.; Garrido, C. and Martínez, V. (2006). Changes in the contents of antioxidants compounds in pepper fruits at different ripening stages, as affected by salinity. Food Chem., 96: 66-73.

Nicholas, S. and Wheeler, L.W. (2000). Ascorbic acid in plants: Biosynthesis and function. Current Rev. in Plant Sci., 19 (41): 267-290.

Nijjar, G.S. (1985). Nutrition of Fruit Trees, Mrs. Usha Raj Kumar for Kalyani Publishers, New Delhi, India.

Pacheco, A.C.; Cabral, C.; Fermino, É.S. and Aleman, C.C. (2013). Salicylic acid induced changes to growth, flowering and flavonoids production in marigold plants. J. Medic. Plants Res., 7(42): 3158 $-3163$. 
Raja Babo, C.; Vigayalakshini, C. and Mohandass, S. (2005). Evaluation of rice (Oriza sativa L.) genotypes for salt tolerance. J. Food Agric. and Environ., 3 (1): 190-195.

Richards, L.A. (1954). Diagnosis and Improvement of Saline and Alkali Soils. U.S. Dept. Agric. Handbook, 60: 160.

Saeidi-Sar, S.; Abbaspour, H.; Afshari, H. and Yaghoobi, S.R. (2013). Effects of ascorbic acid and gibberellin A3 on alleviation of salt stress in common bean (Phaseolus vulgaris L.) seedlings. Acta. Physiol. Plant., 35: 667-677.

Shabana, A.I.; Shafeek, M.R.; Ahmed, H.I. and Abdel-Al, F.S. (2015). Improving growth, fruit setting, total yield and fruit quality of sweet pepper plants (Capsicum annum L.) by using antioxidant and seaweed extracts. Middle East J. Agric. Res., (4): 154-161.

Smirnoff, N. and Wheeler, G.L. (2000). Ascorbic Acid in Plant. Critical Rev. Plant Sci., 19 (4): 267-290.

Snedecor, G.W. and Cochran, W.G. (1980). Statistical Methods $7^{\text {th }}$ Ed. Iowa State Univ., Press. Ame. Iowa, USA.

Sreenivasulu, N.; Grimm, B.; Wobus, U. and Weschke, W. (2000). Differential response of antioxidant compounds to salinity stress in salt-tolerant and saltsensitive seedlings of foxtail millet (Setaria italica). Physiol. Plant., 109: 435-440.
Sumalan, R. and Carmen, D. (2002). Fiziologie vegetala-lucrari practice, Editura Marineasa, Timisoara, 106.

Szepesi, A.; Csiszar, J.; Bajkan, S.; Gemes, K.; Horvath, F.; Erdei, L.; Deer, A.K.; Simon, M.L. and Tari, I. (2005). Role of salicylic acid pretreatment on the acclimation of tomato plants to salt- and osmotic stress. Acta Biol. Szegediensis., 49: 123-125.

Wada, L. and Ou, B. (2002). Antioxidant capacity and phenolic content of Oregon cranberries. J. Agric. Food Chem., 50: 3495-350.

Wassel A.H; Hameed, M.A.; Gobara, A. and Attia, M. (2007). Effect of some micronutrients, gibberellic acid and ascorbic acid on growth, yield and quality of white Banaty seedless grapevines, Afr. Crop Sci. Conf. Proc., 8: 547-553.

Yalpani, N.; Enyedi, A.J.; Leon, J. and Raskin, I. (1994). Ultraviolet light and ozone stimulate accuumulation of salicylic acid, pathogen- related proteins and Virus resistance in tobacco. Plant. 193: pp. 372-376. (Abstract+ References in Scopus/ cited By in Scopus).

Yildırım, E. and Dursun, A. (2009). Effect of foliar SA applications on plant growth and yeild of tomato under greenhouse conditions. Acta Hort. (ISHS), 807: 395-400.

Zhang, G.Y.; Zhao, R.L. and Peng, X. (1999). Oxalate- induced resistance of muskmelon to WMV-2., Chinese Science Bulletin., 44: 1794-1797. 


$$
\text { الملخص العربي }
$$

تحسين إنتاجية القلقل الحلو باستخدام بعض مضادات الأكسدة تحت ظروف الملوحة بجنوب سيناء:

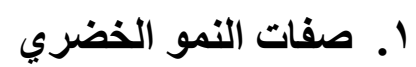

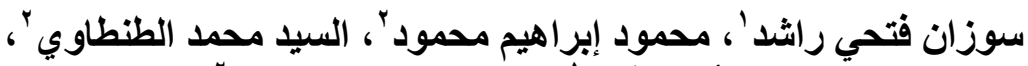

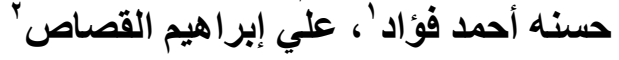

$$
\text { ا ب. بركز بحوث الصحر اء، القاهرة، مصر. }
$$

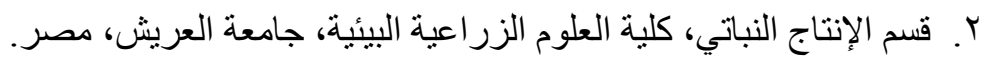

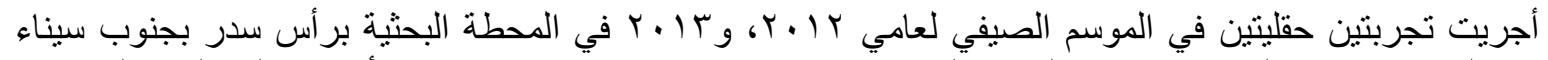

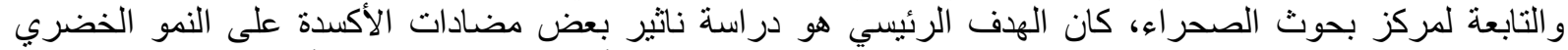

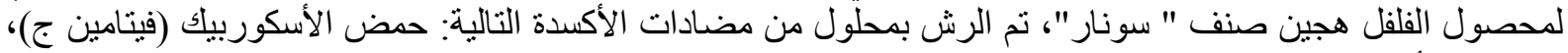

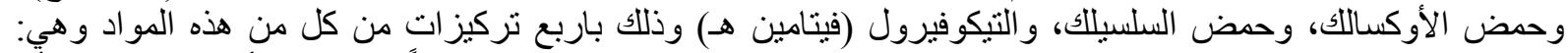

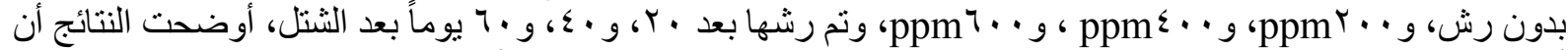

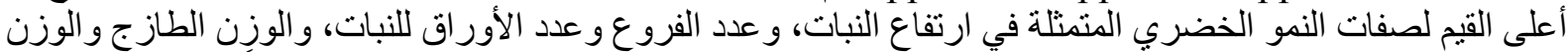

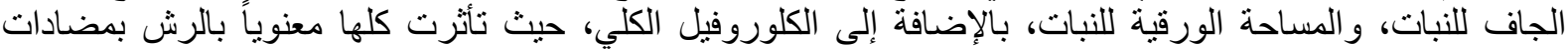

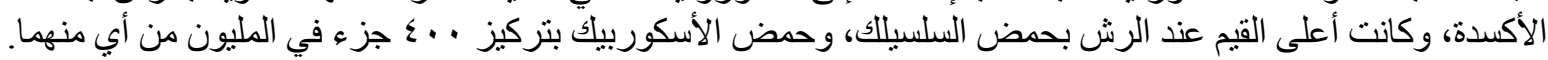

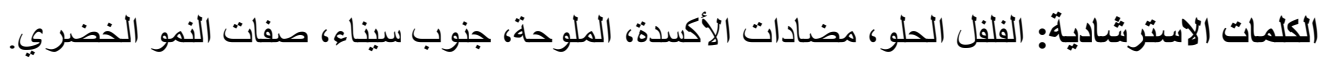


